\title{
Editorial for EAIT issue 2, 2022
}

\author{
Arthur Tatnall ${ }^{1}$
}

Accepted: 1 March 2022/Published online: 7 March 2022

(c) The Author(s), under exclusive licence to Springer Science+Business Media, LLC, part of Springer Nature 2022

Education and Information Technologies (EAIT) is a research journal that covers the complex relationships between Information and Communication Technologies and Education. EAIT is the official journal of the International Federation for Information Processing (IFIP), Technical Committee on Education (TC3).

The first issue of EAIT was published in 1996, making 2020 its twenty-fifth year. To celebrate a quarter-century of EAIT's publication the first article in this issue: Twenty-five years of the Education and the Information Technologies journal: Past and future comes from two of its editors, Arthur Tatnall (Victoria University, Melbourne, Australia) and Andrew Fluck (University of Tasmania, Launceston, Australia). From 1996-2020 (25 years), 1511 articles were published in EAIT. Over this period the journal has grown considerably in size with Volume 1 having a total of only 15 articles while Volume 25 had 268 published articles. With EAIT as an example, the article examines how research publications in education and information technologies have changed over the last twenty-five years. Using Historical Research methodology, the article illustrates some of the trends in topics covered and interrogates the expanding cultural background of published authors. While computer technology has developed rapidly over this time, research methodologies, international collaborations, and discipline areas have multiplied quickly. The article also identifies some unique papers that have appeared in print and provides glimpses into what the future may hold.

David John Lemay (McGill University, Montreal, Canada), Ram B. Basnet (Colorado Mesa University, Grand Junction, USA) and Tenzin Doleck (Simon Fraser University, Burnaby, Canada) then present: Switching intentions in the context of open-source software movement: The paradox of choice. The open-source software movement with software including the Linux-based operating systems, presents a viable alternative to commercial operating systems. This article looks at how the open-source movement might influence user technology switching intentions, specifically to switch to a Linux-based open-source operating system. The article

Arthur Tatnall

Arthur.Tatnall@vu.edu.au

1 Victoria University, Melbourne, Australia 
uses partial least squares modelling to examine the influence of inertia, benefit loss costs, incumbent systems habit, procedural switching costs, sunk costs, social norms, and uncertainty costs, on perceived need and behavioural intention. Overall, the authors found that the results support inertial effects and the influence of social norms on perceived need and users' switching intentions.

The Effect of Augmented Reality based applications on achievement and attitude towards science course in distance education process, from Hakan Çetin and Azmi Türkan (Siirt University, Turkey), describes a study that aimed to enrich a theme in the science course in distance education, with augmented reality-based applications, and to examine the effects of these applications on students' achievement and attitudes in science courses. They found that the students' achievement and attitudes towards the science course increased significantly with Augmented Reality based applications.

Silvia Farias-Gaytan (Tecnologico de Monterrey, Mexico), Ignacio Aguaded (University of Huelva, Andalucia, Spain) and Maria-Soledad Ramirez-Montoya (Tecnologico de Monterrey, Mexico) next write on: Transformation and digital literacy: Systematic literature mapping. Their research aimed to analyse digital transformation and media literacy publications that impact higher education. It aimed to identify the types of research and topics addressed and to explore the scope of digital transformation in higher education institutions. The systematic mapping method was used to analyse published articles in two databases, Scopus and Web of Science (WoS). The research provides a perspective on digital transformation studies in higher education institutions and their internalization approaches.

The next article comes from China, with Yi-Mei Chen (Sanming University, Fujian Province, China) writing on: Understanding foreign language learners' perceptions of teachers' practice with educational technology with specific reference to Kahoot! and Padlet: A case from China. It reports on a classroom-based investigation into English as a foreign language (EFL) learners' views on lessons which integrated m-learning tools for assessment (Kahoot!) and collaboration (Padlet). Chinese university students' views on such lessons were collected through openended questions posted on Padlet. The questions were designed without mentioning the use of these m-learning tools and the data analysed with the combination of comparative content analysis and thematic coding. Major themes emerging from the qualitative data are related to either strategies for teachers to promote learner's motivation or a prerequisite for learning. A striking result is that their perceptions of gamification were amplified when the reality was that only a few class activities used these tools.

The next paper reports on a mixed-methods study of the Total Reading Approach for Children Plus (TRAC+) program, with a particular focus on its mobile learning element, where tablets and an app called Aan Khmer (Read Khmer) were introduced into Cambodian schools to assist 'struggling students' in Grades 1 to 3 learn early reading. Mobile learning for early reading in Cambodia by Grace Oakley and Mark Pegrum (University of Western Australia, Perth, Australia), Thida Kheang (University of Melbourne, Australia) and Krisna Seng (Phnom Penh, Cambodia) focuses primarily on survey and interview data collected in 2017 from relevant staff members, namely school directors, literacy coaches, teachers, and librarians, about 
this intervention program. Findings indicate that staff members were positive about the program, including the app, and observed improvements in the children's reading. Their paper offers insights into the importance of alignment between program design and user readiness, which may inform other technology-based programs intended to raise literacy standards in low- and middle-income countries.

E-Learning has been massively used in higher education institutions, one of which is in Universitas Indonesia write Arief Ramadhan (Bina Nusantara University, West Jakarta, Indonesia), Achmad Nizar Hidayanto, Ghina Almira Salsabila, Intan Wulandari, Jessica Alexia Jaury and Nadia Nabila Anjani (Universitas Indonesia, Jakarta, Indonesia) in: The effect of usability on the intention to use the e-learning system in a sustainable way: A case study at Universitas Indonesia. The e-learning system in Universitas Indonesia is called Student Centered E-Learning Environment Universitas Indonesia (SCELE UI) and the reported research determines the effect of usability on the intention to use SCELE UI in a sustainable way. The Expectation Confirmation Model (ECM) is used as a basis of the research model. On the theoretical side, this research proposes and examines integration of usability and ECM in the context of sustained e-learning usage intention. On the practical side, it also provides insights for developers and universities that can help them to effectively foster the improvement of their system that can ensure sustained usage by their students.

Automatic recommendation system based on hybrid filtering algorithm describes research by Sunny Sharma (University of Jammu, India), Vijay Rana (GNA University, Punjab, India) and Manisha Malhotra (Chandigarh University, Punjab, India). They argue that web recommendation systems are ubiquitous and are used to overcome the product overload on e-commerce websites. In their paper they propose a hybrid system-based book recommendation system that anticipates recommendations. The proposed system is a mixture of collaborative filtering and content-based filtering which can be explained in three phases. In the first phase, it identifies the users who are analogous to the active user by matching users' profiles. In the second phase, it chooses the candidate's item for every similar user by obtaining vectors Vc and Vm corresponding to the user's profile and the item contents. After calculating the prediction value for each item using the Resnick prediction equation, items are suggested to the target user in the final phase.

Although using machine learning for predicting which students are at risk of failing a course is indeed valuable, how can we identify which characteristics of individual students contribute to their being At-Risk? ask Bevan I. Smith, Charles Chimedza and Jacoba H.Bührmann (University of the Witwatersrand, Johannesburg, South Africa) in: Individualized help for at-risk students using model-agnostic and counterfactual explanations. They propose use of local model-agnostic and counterfactual explanations to attack the challenge of characterising individual AtRisk students, and local model-agnostic LIME and SHAP methods were critically evaluated in their study.

A comparative study of computer and mobile-assisted pronunciation training: The case of university students in Taiwan, by En-Minh Lan (National Penghu University of Science and Technology, Taiwan) suggests that English pronunciation training for real-world communication tasks is crucial in EFL learning because it is essential to learners' listening comprehension and speaking competence. 
Computer-assisted pronunciation training (CAPT) has begun to receive more recognition in academic institutions for pronunciation learning, but doubt still remains in regard to its effectiveness in pronunciation and learners' perception. In the reported study, the researcher aimed to explore the effects of mobile-assisted pronunciation training (MAPT) on L2 learners' pronunciation performance.

The next article introduces two new software tools, Bongojontro and Bongojontro Baksobandi, aimed at reducing the barriers to programming for native speakers of Bengali, the fifth most spoken language in the world. Introduction of two new programming tools in Bengali and measurement of their reception among high-school students in Purba Bardhaman, India with the prototypic inclusion of a vector-biology module was contributed by Bishnu Goswami (The University of Burdwan, West Bengal, India) and Sarmila Pal (Hooghly Mohsin College, West Bengal, India). They say that highlights of these software include programming in the native language of Bengali, simpler construction of programs, friendly for beginners, and the possibility of creating and using modules which can be used to incorporate a level of abstraction that can be helpful for users of different technical skills and roles. The prototype vector-biology module, which was a part of Bongojontro Baksobandi, received favourable reviews.

Nazlı Aksu (Hereke Nuh Çimento Middle School, Körfez/Kocaeli, Turkey) and Y1lmaz Zengin (Dicle University, Diyarbakır, Turkey) then offer: Disclosure of students' mathematical reasoning through collaborative technology-enhanced learning environment. The participants of this study were six middle school students, and data consisted of participants' written productions, dynamic materials, and the transcriptions of the participants' discourse. Their analysis showed that the integration of dynamic mathematics software into the ACODESA method (ACODESA is an acronym for the French translation of "collaborative learning, scientific debate and self-reflection') contributes to their collective mathematical reasoning productively, and that the mediational role of dynamic mathematics software also helps them to make dynamic connection between mathematical reasoning and proving.

Virtual Periodic Table (VPT) is an effective software tool to present elements in a conceivable three-dimensional mode say Numan Ali (University of Malakand, Chakdara, Pakistan and The University of Agriculture Peshawar, Pakistan), Sehat Ullah (University of Malakand, Chakdara, Pakistan) and Muhammad Raees (University of Malakand, Chakdara, Pakistan) in their article: Interactive cube for effective demonstration of virtual periodic table. VPT is used to assist users in their learning before performing any hands-on activities in chemistry laboratory. Various VPTs have been developed for enhancing the learning process of chemistry education. Different types of 2D (two-dimensional) interaction interfaces based on menus, buttons, icons etc. are used in the existing VPTs, but the unrealistic 2D nature of these interfaces affects the exploration of chemical elements and the learning performance of students. In their paper, they present a Cube based Interface in Virtual Periodic Table (CIVPT) for demonstration of the detail information of chemical elements. Outcomes of the evaluations revealed that CIVPT enhances students' learning and their motivations in gaining knowledge about chemical elements.

Contradictions in mathematical modelling with digital technologies by Juan Fernando Molina-Toro, Jhony Alexander Villa-Ochoa and Paula Andrea 
Rendón-Mesa (Universidad de Antioquia, Medellín, Colombia) presents an analysis of contradictions expressed by pre-service mathematics teachers when performing two modelling tasks using their own technological devices. Technology and modelling tasks were designed and tested with 14 participants in a mathematical modelling course offered to pre-service teachers in a Colombian university, and linguistic cues were used to carry out a thematic analysis for identifying contradictions in discursive manifestations. Their results show how specific contradictions in modelling processes with technology are manifested by students and the kind of transformations they promote, as well as the changes that occur in how tasks are performed, and how students develop an idea of technology that goes beyond its traditional conception as a tool.

With the rapid technological advancements, schools and teachers have great responsibilities to educate students regarding technological transformations says Mustafa Aydin (Necmettin Erbakan University, Konya, Turkey) in: A multilevel modelling approach to investigating factors impacting computer and information literacy: ICILS Korea and Finland sample. Students' ease of access to ICT tools provides ample opportunities for the development of these skills, not solely limited to schools, and schools invest efforts to contribute to this process through their updated curricula. Teachers' behavioural patterns also contribute to students' learning. In this study, the effect of teacher and student characteristics on students' achievement regarding digital skills were examined together. The analysis involved six models addressing teacher and student characteristics in different contexts. Demographic features, ICT usage purposes and affective characteristics related to ICT were added to the models related to teacher and student characteristics in sets.

Chinese Adolescents' Rebellion during the COVID-19 Pandemic: Discipline and Resistance in Online Compulsory Education by Jindong Liu, Biying Wu and Jiayu Qu (The Chinese University of Hong Kong, Hong Kong SAR, China) tackles the debate surrounding the tension between knowledge and power in online education for adolescents, between freedom and control at large, and how disciplinary power was exercised and resisted in a Chinese setting of online compulsory education during the COVID-19 outbreak in 2020. In the study, 60 participants including Grade 7 to 12 students, their parents, and teachers joined in focus groups or individual interviews in a secondary school in Xi'an, China. By following Foucault's concepts of three techniques of disciplinary power: hierarchical observation, normalizing judgement and examination, they identified four themes based on the data: (1) diminished discipline with the dissolving boundary, (2) reconfigured disciplinary power by teachers, (3) self-discipline as a vital skill, and (4) online compulsory education as a future trend. Interpretations from the Foucauldian perspective are presented, suggesting that most adolescents depend upon more external disciplines from schools and teachers, while only a few may achieve autonomy through self-discipline.

The technological developments of the twenty-first century have enabled the emergence of alternative teaching-learning models and instructional tools. Mustafa Güler (Trabzon University, Turkey) \& Suphi Önder Bütüner (Yozgat Bozok University, Turkey), Şahin Danişman (Düzce University, Turkey) and Kadir Gürsoy (Trabzon University, Turkey) enlarge on this in their article: A meta-analysis of the 
impact of mobile learning on mathematics achievement. The aim of their study was to test the effect of mobile learning on students' mathematics achievement by undertaking a systematic database search that included the Academic Search Complete, Education Resources Information Center (ERIC), EBSCO, JSTOR, ScienceDirect, Taylor \& Francis Online, and Web of Science to examine peer-reviewed articles that met the inclusion criteria. The results show that mobile learning has a medium-level positive effect on students' mathematics achievement.

Mathematics learning and assessment using MathE platform: A case study describes research by Beatriz Flamia Azevedo (Instituto Polit'ecnico de Bragança, Portugal and University of Minho, Portugal), M.FatimaPacheco (Instituto Polit'ecnico de Bragança, Portugal and University of Aveiro, Portugal), AnaI. Pereira (Instituto Polit'ecnico de Bragança, Portugal and Portugal and University of Minho, Portugal) and Florbela P. Fernandes (Instituto Polit'ecnico de Bragança, Portugal). Universities are encouraging the implementation of innovative methodologies and teaching strategies to develop an interactive and appealing educational environment where students are the focus of the learning process. MathE has been developed to help achieve this goal and to nurture and stimulate the learning of Mathematics in higher education. This study introduces and describes the MathE platform, which is divided into three sections: Student's Assessment, Library, and Community of Practice. The results indicate the platform is well organized, with a satisfactory amount and diversity of questions and good interconnection between the various parts.

Seyyed Morteza Hashemi Toroujeni (Iran Ministry of Education, Chabahar, Konarak, Iran) then presents: Computerized testing in reading comprehension skill: investigating score interchangeability, item review, age and gender stereotypes, ICT literacy and computer attitudes. Score interchangeability of Computerized Fixed-Length Linear Testing (CFLT) and Paper-and-Pencil-Based Testing (PPBT) has become a controversial issue over the last decade when technology has meaningfully restructured methods of educational assessment, and various testing guidelines published on computerized testing may be used to investigate the interchangeability of CFLT and PPBT mean scores to corroborate if test takers' testing performance is influenced by the effects of testing administration mode. This research was conducted to probe not only score interchangeability across testing modes but also the role of age and gender stereotypes, item review, ICT literacy and attitudes towards computer use as moderator variables in test takers' reading achievement in CFLT. Findings demonstrated that although the reading scores of test takers were interchangeable in both CFLT and PPBT versions regarding testing administration modes, they were different regarding item review, and no significant interaction was found between age, gender, and ICT literacy and CFLT performance.

Improving OER descriptions to enhance their availability, reuse, and enrichment was contributed by Jhon Francined Herrera-Cubides, Paulo Alonso Gaona-García and Carlos Enrique Montenegro-Marín (Universidad Distrital Francisco José de Caldas, Bogotá, Colombia) and Salvador Sánchez-Alonso (Universidad de Alcalá de Henares, Spain). Information and communication technologies and virtual training have increased the use of educational resources. Resource descriptions adapted to particular needs and lack of metadata enrichment taking advantage 
of benefits provided by the Semantic Web are some examples of problems. The purpose of this paper is to expose an enhanced and interoperable set of metadata elements for describing OER (Open Educational Resources), which takes full advantage of the Openness and data enrichment.

The shift towards digitalization in education, intensified by COVID-19 pandemicrelated issues, has led to the emergence of a need to expand the borders of the teaching profession, and Evgenia A. Yurinova, Olga G. Byrdina and Svetlana G. Dolzhenko (University of Tyumen, Ishim, Russia) investigate this in: Transprofessional competences of school teachers in the digital environment: education employers' perspective. To be effective in the digital environment, school teachers have to master new transprofessional competences (TPCs), enabling them to use the skill sets of other careers, gain new skills and abilities and find non-standard solutions to professional and pedagogical problems using digital technologies. This study investigated education employers' opinions (61 principals and deputy principals from 31 Russian secondary schools) about the comparative importance of TPCs for school teachers working in the digital environment and their perceptions of what barriers prevent teachers from developing TPCs. The findings showed that employers attached the greatest importance to psychological TPCs while assigning the least importance to digital citizenship TPCs. The major barriers to TPC development, in the respondents' opinion, lay in teachers' fear of new trends and their excessive workload.

Clifford Agyei and Özge Razi (Cyprus International University, Nicosia, Turkey) then offer: The effect of extended UTAUT model on EFLs' adaptation to flipped classroom. They suggest that educational transformation of flipped classroom continues to be the important approach to increasing students' readiness for flipped learning. To successfully implement the flipped classroom, students' readiness to use the materials delivered to them in the pre-class session and parent-school involvement should be part of the process to improve this student-centred learning approach, but little is known concerning the assessment of students' readiness to learn through Web-based Instruction (WBI) in a flipped classroom and the role of parent-school involvement in student-centred learning approach. The results of their work showed that performance expectancy, effort expectancy, parent-school involvement, perceived self-efficacy and experience expectancy have positive influence on students' behavioural intention to use WBI.

Stylianos Mystakidis (University of Patras, Rion Achaia, Greece), Athanasios Christopoulos (University of Turku, Finland) and Nikolaos Pellas (University of Western Macedonia, Greece) argue that while there is an increasing interest in Augmented Reality (AR) technologies in Primary and Secondary Education, its application in Higher Education is still an emerging trend. This study: A systematic mapping review of augmented reality applications to support STEM learning in higher education reports findings from a systematic mapping review, based on a total of forty-five articles published in international peer reviewed journals from 2010 to 2020, after evaluating the use of AR applications that support Science, Technology, Engineering and Mathematics (STEM) subjects' learning in HE settings.

Identification and evaluation of technology trends in K-12 education from 2011 to 2021 is by Adam Kenneth Dubé and Run Wen (McGill University, Montreal, Canada). They note that educational technologies have captured the 
attention of researchers, policy makers, and parents and considerable effort and money are invested into new technologies, hoping to find the next effective learning tool. This paper provides an overall picture of the changing trends in educational technology by analysing the Horizon Reports' predictions of the most influential educational technologies from 2011 to 2021, identifying larger trends across these yearly predictions, and by using bibliometric analysis to evaluate the accuracy of the identified trends.

Sacip Toker (Atilim University, Ankara, Turkey) next writes on: The progress of twenty-first century skills throughout instructional design projects: a quasi-experimental comparison of rapid prototyping and Dick and Carey models. The study investigates this using a causal-comparative design. The participants were computer education and instructional technology department students who registered for a core instructional design course and were divided into two groups: Rapid prototyping model and Dick and Carey model. Results showed that the participants demonstrated an increase in time management skills and a decrease in cooperation process skills in both groups.

Forecasting the enrolments of new students in bachelor's systems has become urgent in many higher education institutions and represents an important stage in the process of making strategic decisions for new course's accreditation and optimization of resources. In Forecasting approaches in a higher education setting, Hassan Bousnguar, Lotfi Najdi and Amal Battou (Ibn Zohr University, Agadir, Morocco) investigate this by looking at the most used machine learning and statistical approaches. The main result of this study is the development of a forecasting model that provides the most accurate values with a minimum of errors.

Effects and acceptance of precision education in an AI-supported smart learning environment by Yung-Hsiang Hu (University of Science and Technology, Taiwan) presents precision education that aims to regulate students' behaviours through the learning analytics dashboard (LAD) in the AI-supported smart learning environment (SLE). The LAD basically tracks and visualizes traces of learning actions to make students aware of their learning behaviours and reflect these against the agreed goals. This research aimed to realize the digital transformation of the learning space, thereby improving students' learning outcomes with the assistance of the learning dashboard.

Using $Q R$ code enhanced authentic texts in EFL extensive reading: a qualitative study on student perceptions, is by Safiye İpek Kuru Gönen (Anadolu University, Turkey) and Gülin Zeybek (University of Applied Sciences, Turkey). They point out that increased use of recent technological devices by language learners at all ages and various levels stimulates the integration of the latest technological devices into the language learning process. Considering the numerous advantages of augmented reality (AR) in creating a supportive learning environment, this study aimed at investigating the use of quick response codes, as an AR feature, during extensive reading. For the study, QR code enhanced authentic reading texts were designed to aid learners in the comprehension of culture-specific terms that were difficult to be understood with a dictionary definition. Findings underline the potential of integrating AR technology such as QR codes into language education for more effective learning opportunities and highlight the 
importance of providing access to technology in order to help all students benefit from its ubiquitous nature.

A systematic literature review regarding computational thinking and programming in early childhood education by Kaan Bati (Hacettepe University, Ankara, Turkey) aimed at summarizing experimental evidence regarding computational thinking and programming conducted in early childhood education in terms of the variables of plugged-in versus unplugged, age, and gender. WoS, Scopus, and Eric databases were scanned, and studies determined to be within the scope of the systematic scanning criteria were selected for review. The study found that age was an important factor in learning computational thinking in early childhood, girls and boys performed similarly in programming and computational thinking, and although there was concrete evidence that both plugged-in and unplugged applications improved children's computational thinking skills, it appeared that unplugged applications were one step ahead, considering the power of having concrete experiences.

The next article is of a very different topic: The effectiveness of video prompts created with animations in teaching car wash skills. It comes from Hasan Köse and Mine Sönmez-Kartal (Eskisehir Osmangazi University, Eskişehir, Turkey). This study aimed to investigate the permanence of the teaching, the generalizability of the acquired skill, and the opinions of the participants and their families in research about animation video prompting (VP), and teaching car wash skills to individuals with mild intellectual disability (ID). The participants of the study were three males with mild ID, ages 18-24 and the findings showed that individuals with mild ID can learn the car wash skill using the VP method prepared with animations. The use of animation can facilitate the preparation of video-based content.

André Menolli (Universidade Estadual do Norte do Paraná-UENP, Brazil and State University of Londrina-UEL, Brazil) and João Coelho Neto (Universidade Estadual do Norte do Paraná-UENP, Brazil) then write on Computational thinking in computer science teacher training courses in Brazil: A survey and a research roadmap. This work aims to understand how computational thinking is addressed, and which digital technologies and teaching methods are used to foster it. Their main results are: common and accessible technologies used in everyday life can help promote computational thinking; researchers and teachers can explore the list of technologies surveyed and categorized to promote computational thinking; teachers can analyse the teaching methods used and understand how these methods are applied in the teaching process.

Effect of instructional methods on pre-service science teachers learning outcomes: a meta-analysis by Josiane Mukagihana and Florien Nsanganwimana (University of Rwanda-College of Education, Rwamagana, Rwanda) and Catherine M. Aurah (University of Science and Technology, Kakamega, Kenya) remarks that best instructional methods are essential tools to bring desirable changes in preservice science teachers, but that available literature lacks enough meta-analytic review illustrating the effect of various instructional methods on pre-service science teachers' learning outcomes. Their meta-analytic review identified instructional methods used for teaching pre-service science teachers the period from 2010 to 2020. Fieldbased inquiry focused model of instruction, and documentary films-based instruction were determined to be the most effective than traditional methods. 
COVID-19, emergency remote teaching evaluation: the case of Indonesia is from Ani Cahyadi (Universitas Islam Negeri Antasari, Banjarmasin, Indonesia), Hendryadi (Sekolah Tinggi Ilmu Ekonomi Indonesia Jakarta, Indonesia), Sri Widyastuti (Universitas Pancasila, Jakarta, Indonesia) and Suryani (Universitas Islam Negeri Sultan Maulana Hasanuddin Banten, Indonesia). They point out that the global crisis caused by the COVID-19 pandemic has challenged educational institutions worldwide to rapidly shift to an online mode of teaching, and in their paper, they discuss the concept of emergency remote teaching (ERT), including its implementation and evaluation, in the context of higher education in Indonesia. The Context, Input, Process, and Product framework was used to evaluate the implementation of ERT based on the experiences of 45 faculty members and 82 students from seven universities and colleges in three provinces in Indonesia. This study provides recommendations that they say, will serve as input for future strategies and educational policies in Indonesia, and developing countries in general.

In the next article, Hana Vonkova, Ondrej Papajoanu and Katerina Kralova (Charles University, Prague, Czech Republic) argue that with the current emphasis placed on ICT skills development in education, accurate information about how well students master these skills becomes invaluable, but despite the wide-spread use of self-report measures of ICT skills, their accuracy has been questioned. Student online communication skills: Enhancing the comparability of self-reports among different groups of students investigates this issue as the authors claim that an analysis, on a large sample, of the heterogeneity in reporting behaviour in the domains of ICT competencies is missing. They investigate the (in)comparability of self-reports of online communication skills, such as using of social networks and data sharing, among two contrasting groups of students (a) elite, high-performing grammar schools and (b) economics schools.

Mohammad Arfaee (Islamic Azad University, Tehran, Iran), Arman Bahari (University of Sistan and Baluchestan, Zahedan, Iran) and Mohammad Khalilzadeh (Pontificia Universidad Católica del Perú, Lima, Peru) then write on: A novel prediction model for educational planning of human resources with data mining approach: a national tax administration case study. They point out that human resources training is considered an effective solution in empowering human resources, and that organisations try to have effective educational planning for this precious resource by identifying shortcomings through a need assessment. This study provides a model based on organisational data analysis to achieve a unique and appropriate training planning for each staff.

Thasnim Humida (Begum Rokeya University, Rangpur, Bangladesh), Md Habib Al Mamun and Pantea Keikhosrokiani (Universiti Sains Malaysia, Penang, Malaysia) suggest that digital transformation and emerging technologies open a horizon to a new method of teaching and learning and revolutionises the e-learning industry. Their study: Predicting behavioural intention to use e-learning system: A case-study in Begum Rokeya University, Rangpur, Bangladesh scrutinises a proposed research model for predicting factors that influence student's behavioural intention to use e-learning system at Begum Rokeya University, Bangladesh. The study used quantitative approach and developed a research model based on several technological acceptance models. 
The next study, by Anna Shmakova (Ulyanovsk State Pedagogical University, Ulyanovsk, Russian Federation), Yulia Ryzhova (State University of Management, Moscow, Russian Federation) and Alexey Suhorukhih (Voronezh State Forestry Engineering University Named After G. F. Morozov, Voronezh, Russian Federation): The impact of ICT education on humanistic innovative potential develops an analytical framework to study the effect of ICT education on humanistic innovative potential. The study recruited 150 school age children from three secondary schools and 100 university students from two universities in Russia and each respondent's humanistic potential was measured using the Pomitkin's Spiritual Potential of a Personality Inventory. The proposed framework consists of several components: a motivational component, a content component, an activity component, a value component, and an imitational component The results of the study show that the proposed framework allows improving the spiritual potential of learners.

Experiencing the community of inquiry framework using asynchronous online role-playing in computer-aided instruction class by Kasiyah Junus, Harry Budi Santoso and Mubarik Ahmad (Universitas Indonesia, Jawa Barat, Indonesia) investigates the use of online role-playing, in an online discussion forum, in learning the community of inquiry framework - an area of learning covered in the ComputerAided Instruction course, an elective course for Computer Science undergraduate students at Universitas Indonesia. The participants were divided into different roles and each group was triggered to discuss the implementation of online collaborative learning. Teaching presence was the rarest, as students were focused on delivering their ideas. Social presence appeared in almost all messages since it is the easiest, and students can feel the impact immediately.

Elsa Costa e Silva, Teresa Lino-Neto, Eugénia Ribeiro, Miguel Rocha and Manuel João Costa (University of Minho, Portugal) then offer: Going virtual and going wide: comparing Team-Based Learning in-class versus online and across disciplines. Team-based learning (TBL) is an active learning pedagogy developed for in-class sessions and based on the collaborative work of small groups of students. The increasing push to online and blended learning has enhanced the need to expand this pedagogy to a virtual environment, but little evidence has been produced on how students accept online synchronous sessions of TBL. This study presents a comparative perspective of traditional in-class versus adapted fully synchronous online TBL and across different disciplinary fields. Results obtained from this study revealed a wide approval of TBL, regardless of the online or in-class TBL sessions environment, scientific area of courses and student gender.

The next study, by Y1lmaz Zengin (Dicle University, Diyarbakır, Turkey) focused on how university students constructed proof of the Fundamental Theorem of Calculus (FTC) starting from their argumentations with dynamic mathematics software in collaborative technology-enhanced learning environment: Construction of proof of the Fundamental Theorem of Calculus using dynamic mathematics software in the calculus classroom. The data consisted of participants (university students) written productions, dynamic materials, and the transcriptions of the participants' argumentations for the selected groups and analysis was based on the integration of $\mathrm{cK} \phi$ model and Toulmin's model. The analysis showed that the collaborative technology-enhanced learning environment helped the participants to interpret the Mean 
Value Theorem (MVT) for definite integrals geometrically and use this interpretation for the proof of the FTC.

A card game for designing activities for technology-enhanced learning in higher education was contributed by Anna Mavroudi (Norwegian University of Science and Technology, Trondheim, Norway), Teresa Almeida (Umeå University, Sweden), Susanne Frennert (Malmö University, Malmö, Sweden), Jarmo Laaksolahti and Olga Viberg (KTH Royal Institute of Technology, Stockholm, Sweden). They point out that the importance of providing mechanisms and tools that effectively support the transition from implicit to explicit representations of Learning Design has been emphasised by previous research in the field of TechnologyEnhanced Learning (TEL), and the benefits of Game-based learning approaches have been long documented in the educational research literature. This paper presents the design, implementation and evaluation of a card game that aims to support the design process of TEL activities in higher education. Results revealed that the participants a) are satisfied with the game process, b) appreciate the groupwork and interaction taking place, and c) believe that they used their communication and collaboration skills.

Medical and public health instructors' perceptions of online teaching: A qualitative study using the Technology Acceptance Model 2 is by Meina Zhu (Wayne State University, Detroit, USA) and Yu Zhang (Farmington Hills, MI, USA). Many universities in the U.S. shifted from in-person teaching to online teaching due to the COVID-19 pandemic. Instructors' acceptance of online teaching plays a crucial role, as the acceptance level can impact instructors' online teaching behaviours. This qualitative study examined medicine and public health instructors' perceptions of online teaching using the Technology Acceptance Model 2 (TAM2) model. Through semi-structured interviews with ten instructors in a Midwest university in the U.S., this study found that instructors had a high level of acceptance of online teaching. They perceived the usefulness of online teaching in terms of learning objectives, assessment, instructional methods, and learning experience.

The purpose of the next research study, by Kubra Karakaya-Ozyer and Zeki Yildiz (Eskisehir Osmangazi University, Eskisehir, Turkey): Design and evaluation of an electronic performance support system for quantitative data analysis was to develop this system by using design-based research. In the first phase of the research, the tendencies, challenges, and problems of educational researchers in terms of quantitative research were identified. Next, a prototype of the Electronic Performance Support System (EPSS) for quantitative data analysis was designed and then the EPSS was tested and evaluated in three cycles. The results of the evaluation phase showed that the system proved to be useful to solve the problems encountered during the quantitative data analysis process.

Tiphaine Colliot (Université de Poitiers, France), Kenneth A. Kiewra (University of Nebraska-Lincoln, USA), Linlin Luo (University of Regensburg, Germany), Abraham E. Flanigan (Georgia Southern University, Statesboro, USA), Junrong Lu, Carrie Kennedy and Sheldon Black (University of Nebraska-Lincoln, USA) next write on: The effects of graphic organizer completeness and note-taking medium on computer-based learning. Their study aimed to determine how graphic organizer completeness (complete, partial, or no organizer) and note-taking medium 
(longhand or computer) affect note-taking quantity, quality and computer-based learning. College students were presented with a computer-based PowerPoint lesson accompanied by complete, partial, or no graphic organizers. Throughout the lesson, students recorded notes using either longhand or computer mediums and were immediately tested and again two days later following a review period during which graphic organizers and notes were studied. Results suggested that complete organizers aid germane load more than partial organizers and that longhand note taking results in deeper processing than does computer note taking.

A meta-analysis and bibliographic review of the effect of nine factors on online learning outcomes across the world is by Zhonggen Yu (Beijing Language and Culture University, China) who points out that the unpredictable pandemic has drastically altered learning approaches, where online learning has been booming. Through VOSviewer, this study visualizes the network of top authors, organizations, sources, and countries that have been devoted to online learning. This study identifies nine variables that may exert a significant effect on online learning outcomes during this special pandemic time in various countries and areas.

Zahra Sorat and Zohre Mohamadi Zenouzagh (Islamic Azad University, Karaj, Iran) then write on: Designing and validating an assessment inventory for online language teacher education accountability. They note that education accountability and its building components has been the focal point and yet a convoluted issue, and their study aimed to give a comprehensive account of indicators of education accountability in e-learning. To this end, this two-phase study was conducted in an Iranian English as Foreign Language context. The first phase was qualitative in nature and aimed at identifying the indicators through conversation analysis of stored interviews with distinguished English as foreign language teachers who hold online EFL teacher training courses in three different language centres in Tehran. Open coding and thematic analysis via NVivo software on the interviews made the building blocks of the second phase of this study which was designing and validating a questionnaire for assessing educational accountability in e-learning.

Using mobile applications in science education has proven to be effective and add benefits including learning gains, motivation to learn, and collaborative learning say Hüseyin Ateş (Kırşehir Ahi Evran University, Turkey) and Juan Garzón (Universidad Católica de Oriente, Rionegro, Colombia) in their article: Drivers of teachers' intentions to use mobile applications to teach science. Some teachers, however, are reluctant to use this technology, and it is important to identify what factors affect teachers' intentions to use mobile applications and to encourage them to use this technology in their classes. This study proposes a model to predict science teachers' intentions to use mobile applications in the teaching process. The model merges the Technology Acceptance Model, Flow Theory, and the Theory of Planned Behaviour.

Maryam El Asame, Mohamed Wakrim and Amal battou (Ibn Zohr University, Agadir, Morocco) investigate E-Assessment, as this is a key element in the instructional design process and plays a major role in supporting and enhancing learning: Designing e-assessment activities appropriate to learner's competency levels: Hybrid pedagogical framework and authoring tool. Current e-assessment design, however, does not achieve the desired pedagogical objectives in the e-learning environments and in their paper they propose a hybrid pedagogical framework that 
includes a set of principles for competencies assessment design based on the Competency-based Approach (CBA) and Objective-based Approach (OBA).

Developing an innovator's thinking in engineering education by Anna Solodikhina (National Research University Higher School of Economics, Moscow, Russian Federation) and Maria Solodikhina (MPGU International Relations Office Moscow Pedagogical State University, Moscow, Russian Federation and Peoples' Friendship University of Russia Moscow, Russian Federation) argues that engineering education must be changed following the change in the labour market. The cult of innovation has led to a demand for innovators who have both the mindset of an inventor who can see a problem and find a way to solve it, and the mindset of an entrepreneur who is ready to bring that solution to life and so teaching a technoinnovator involves combining STEM education and business education with a common goal to develop the innovator's thinking as a symbiosis of design thinking and business thinking. The tool for the formation of design thinking can be the link of "STEM cases + project", and the tool for the development of business thinking "entrepreneurial cases + startup", a startup as the inculcation and diffusion of the project product.

Computational thinking (CT) is an essential skill in the twenty-first century and Dewi Muliyati (Universitas Negeri Jakarta, Indonesia), Dadan Sumardani (National Chiayi University, Taiwan), Siswoyo Siswoyo, Fauzi Bakri, Handjoko Permana and Erfan Handoko (Universitas Negeri Jakarta, Indonesia) and Ni Larasati Kartika Sari (Universitas Nasional, Jakarta, Indonesia) expound on this in: Development and evaluation of granular simulation for integrating computational thinking into computational physics courses. The computational physics course (CPC) is one subject that is designed to support students in the practice of CT. Many studies show that the worksheets could be a solution in a CPC as a scaffold to achieve the CT objectives both online and offline and the study develops the worksheet and integrates it with CT in a computational physics course.

An intelligent tutoring system architecture based on fuzzy neural network (FNN) for special education of learning disabled learners by Sarthika Dutt, Neelu Jyothi Ahuja and Manoj Kumar (University of Petroleum and Energy Studies, Uttarakhand, India) notes that several studies have investigated the need for learning difficulties identification specifically Dyslexia, Dysgraphia and Dyscalculia, but identification of these difficulties with children is a multiple screening process under psychologist's supervision and is a difficult task. The introduction of an Intelligent Tutoring System (ITS) to identify learning problems and teach the learning disabled through ITS is worthwhile and integration with AI methodologies have put together promising results. Results indicate that the integration of fuzzy with the neural network has significantly increased the ITS accuracy.

Nina Bergdahl (Stockholm University, Sweden) and Melissa Bond (University College London, UK) note that it is well-recognised that engagement is critical for learning and school success, but that engagement and disengagement are also influenced by context. They tackle this issue in their article: Negotiating (dis-)engagement in K-12 blended learning. As digital technologies add complexity to the educational context, they influence classroom leadership, lesson designs and related practices, and thereby engagement. In this qualitative study, classroom observations 
were conducted in an upper secondary school in Sweden, along with dialogues with teachers and students. Identified themes include digital context, teacher leadership, engagement and disengagement. A network of relations between the (dis-)engagement compound and themes is presented. The results identified processes in which engagement shifted into disengagement and vice versa; in particular, that the intention of active learning does not automatically translate to active learning for all students, although teachers employed a higher work pace than did their students.

Systemic model for technology integration in teaching from Yehuda Peled (Western Galilee College, Akko, Israel) and Sara Perzon (The Open University, Rannana, Israel) investigates the factors influencing Israeli teachers who participated in the national 'Laptop for Every Teacher' program (LET) three-year technology integration program. They assert that although there are several variables influencing the success of such an extensive ICT integration program, school management attitude is crucial, and a key factor for the program's success. The results of their analysis indicate that the variance in attitudes toward technology use is explained by support from management, technology use before the training, and seniority in teaching. They conclude that a top-down initiative forcing the school administration to participate in a long-term process aiming at changing the school's culture cannot succeed without engaging the principals into the program and advancing them to technological leaders, and that without the schools' administration support, teachers are more likely to continue teaching in the method with which they are most familiar.

Educational indicators have revealed that a significant portion of Brazilian Basic Education students have a less than satisfactory skill level in reading and solving mathematics problems, say Sivaldo Joaquim and Ig Ibert Bittencourt (Federal University of Alagoas, Brazil), Patrícia Leone Espinheira (Federal University of Pernambuco, Brazil), Marcelo Reis and Rafael de Amorim Silva (Federal University of Alagoas, Brazil) in: What to do and what to avoid on the use of gamified intelligent tutor system for low-income students. Despite several proven benefits, adaptive learning technologies are scarcely used with low-income students in public schools' due to unavailable resources and lack of technological infrastructure. This study aimed to identify good and bad pedagogical practices from teaching and learning processes using a gamified Intelligent Tutor System (ITS) in Elementary Education. Results observations made in classrooms provided evidence of significant improvement in the domain of Portuguese Language and Mathematical skills.

Aloys Iyamuremye (African Centre for Innovative Teaching and Learning Mathematics and Science, Rwanda), Janvier Mukiza (African Centre for Innovative Teaching and Learning Mathematics and Science, Rwanda and University of Rwanda, Rwanda), Ezechiel Nsabayezu, Fidele Ukobizaba and Kizito Ndihokubwayo (African Centre for Innovative Teaching and Learning Mathematics and Science, Rwanda) then write on: Web-based discussions in teaching and learning: Secondary school teachers' and students' perception and potentiality to enhance students' performance in organic chemistry. Interviews and achievement tests were applied for collecting data. Results from this study revealed that applying web-based discussions is the teaching method that potentially and effectively enhances students' 
performance in organic chemistry, and students and teachers expressed positive perceptions to the application of web-based discussions in teaching and learning.

Mobile device use among preschool-aged children in Greece comes from Stamatios Papadakis, Foteini Alexandraki and Nikolaos Zaranis (University of Crete, Rethymnon, Greece). They note that in the last decade, interactive touchscreen devices have become ubiquitous among young children, and toddlers first experience touchscreen technology before two. Although parents have a vital role in developing the home environment as a stimulus for development, they also have conflicting views on the appropriateness of using apps to deliver educational content. The purpose of the study was to reveal various aspects of children's smart mobile use at home, such as the frequency of mobile device usage, preferred app types, and parent beliefs and strategies. The study showed that parents seek to support their children's learning at home via mobile devices.

Online discussions have become important educational activities through which collaborative learning and knowledge construction can be facilitated, and this study explores the use of two online discussion tools: online discussion board and VoiceThread, for supporting online course discussion activities among a group of graduate students and investigates the differences in the levels of social presence and social knowledge construction in these discussion settings. Investigation on graduate students' social presence and social knowledge construction in two online discussion settings was presented by Chen Guo and Peter Shea (University at Albany, New York, USA) and Xiangdong Chen (East China Normal University, Shanghai, China). The participants' posting behaviours and interaction patterns were also examined, and results showed that participants contributed more words when using the audio and video features of VoiceThread.

A systematic literature review of web-based student response systems: Advantages and challenges describes research by Omer Kocak (Ataturk University, Turkey). Web-based student response systems (SRSs) are one of the Web 2.0 tools that have recently started to be used frequently by researchers and educators, with the widespread use of smartphones among students. The reported study presents a systematic review of the literature on SRS used in educational settings. Findings showed that SRSs was mostly used in undergraduate courses and in the field of medicine. In the articles it was seen that there were generally long-term implementation periods and that the tools Kahoot! and Socrative were preferred most frequently.

From Brazil, Viviane Brito Nogueira, Diego Gomes Teixeira, Ivan Alisson Cavalcante Nunes de Lima, Bárbara Sthéphane Caixeta de Oliveira, Marcus Vinícius Chaves Moreira, Iago Matheus Bezerra Pedrosa, Jose Wilton de Queiroz and Selma Maria Bezerra Jeronimo (Federal University of Rio Grande Do Norte, Natal, Brazil) present: Towards an inclusive digital literacy: An experimental intervention study in a rural area of Brazil. The authors remark that about half of the world's population remains without access to internet in an era of digital transformation. In their study, they investigated the impact of implementing the use of logic and mathematics through digital literacy on a population of elementary school students in a town in Northeast Brazil. In a non-randomized experimental longitudinal intervention study, 5th-grade students were followed during one semester and underwent observational testing during class with the use of scales to evaluate their activities in 
a digital environment. They were evaluated with respect to their ability to use digital devices. Students progressively improved their interaction with the computer (touchpad and typing skills) and their confidence in the digital environment.

Adoption of ICT as a pedagogical tool in community secondary schools in Tanzania: Possibilities and Constraints by Mwema M. Warioba, Haruni Machumu, Koloe Kulunga and Leila Mtweve (Mzumbe University, Morogoro, Tanzania) explores possibilities and constraints for adopting ICT as a pedagogical tool among teachers and students in community secondary schools (CSSs). The study used five CSSs located in rural peripheral in Serengeti District. The results revealed several possibilities for ICT adoption in some of CSSs: presence of electricity; presence of ICT devices; presence of technical support and positive attitude toward ICT use, but several constraints for ICT adoption included insufficient ICT devices: lack of technical support and lack of ICT skills and knowledge. The study findings suggest that teachers and students have positive attitudes towards the technology use.

The last paper in this issue comes from Mahmood H. Hussein and Siew Hock Ow (University of Malaya, Kuala Lumpur, Malaysia), Monther M. Elaish (University of Benghazi, Libya) and Erik O. Jensen (University of Aalborg, Denmark) who write on: Digital game-based learning in K-12 mathematics education: a systematic literature review. They note that application of mathematical skills is essential to our daily routine and is foundational for numerous disciplines. Among various computer-supported learning methods, Digital Game-Based Learning (DGBL) has been perceived as a promising method in teaching mathematics, promoting students' interest, and motivation, and the aim of this systematic literature review was to provide a detailed synthesis of literature regarding the effectiveness of DGBL applications in K-12 mathematics education and extend the findings of previous reviews. Most of the reviewed studies reported positive gains in all categories, with the traditional method of teaching being the most popular comparison approach.

Articles in this issue come from researchers in: Australia, Bangladesh, Brazil, Cambodia, Canada, China, Colombia, Czech Republic, Denmark, France, Germany, Greece, Hong Kong, India, Indonesia, Iran, Israel, Kenya, Libya, Malaysia, Mexico, Morocco, Norway, Pakistan, Peru, Portugal, Russian Federation, Rwanda, South Africa, Spain, Sweden, Taiwan, Tanzania, Turkey, UK, USA.

Dedicated, professional reviewers are an essential part of any research journal and EAIT is fortunate to have some excellent reviewers. We do, however, have a problem. We do not have enough high-quality reviewers! In 2021 we had 2,583 submissions to the journal (352 articles were published). This means a huge effort for our present number of reviewers. We need more people to register as reviewers and we encourage readers to consider registering and inviting their colleagues also to register. To do this, go to the EAIT Editorial Manager (https://www.editorialm anager.com/eait/default.aspx) and click on Register Now.

Publisher's note Springer Nature remains neutral with regard to jurisdictional claims in published maps and institutional affiliations. 